\title{
Effects of Repeated Laundering and Crease-Resistant Treatment on Fabric Properties
}

\author{
O. Shurkian, J. Amirbayat and R. H. Gong* \\ Department of Textiles, University of Manchester Institute of Science and Technology, \\ PO Box 88, Manchester M60 1QD, United Kingdom
}

\begin{abstract}
Laundering is required for many textile products, but it degrades the fabric performance. We have quantitatively investigated the relationship between the number of laundering cycles and several key fabric performance parameters. The effects of crease resistant treatment are also examined.

As expected, increased laundering gradually decreases the fabric strength. However, the fabric abrasion resistance is increased by laundering. This is caused by fabric shrinkage which increases the tightness, thickness and areal density of the fabric. The crease resistant treatment improves the fabric dimensional stability, but reduces the fabric strength.
\end{abstract}

Keywords: laundering, crease resistance, fabric strength, shrinkage

\section{Introduction}

The most common aftercare of a textile product is laundering. However, during laundering, the fabric is subjected to complex thermal, mechanical and physical actions in both wet and dry conditions. These actions inevitably change the properties of the fabric and in most cases lead to deteriorations of fabric performance.

For cotton fabrics, there is clear evidence that repeated laundering significantly weakens their tensile strength but at the same time increases their fluidity $[1,2,3]$. Cotton and viscose, being weaker than polyester, are more prone to damage than the latter during laundering [4]. The laundering conditions including the chemical condition which is defined by the water hardness and the added detergent or other additives, the temperature and also the intensity of the mechanical action all affect how the fabric properties are changed by laundering $[5,6,7,8,9,10]$. Most textile products must be able to withstand a reasonable number of laundering cycles. For shirts, the average life expectancy is approximately 30 to 40 launderings [11, 12]

It is well known that the application of resin to a fabric increases their resistance to creasing and shrinkage during washing. It may also give the fabric a fuller handle and better appearance [13] but the fabric tends to lose some of its water absorbency [14].

In this work, the effects of repeated laundering on the physical properties of shirting fabrics made from cotton/polyester blended yarns, with and without crease resistant treatment, are investigated.

\section{Materials and Methods}

The two fabrics were made from 19 tex yarns of cotton/polyester $(50 / 50)$ blend. They have similar thread density, cover factor and areal density. Crease-resistance treatment was applied to one of the fabrics. The fabric was first impregnated in a solution containing concentrated Fixapret CP (60 g/l) and magnesium chloride (12 g/l). No softeners and wetting agents were used in the formulations. Wet pick up was in the range of $65-70 \%$, based on the original weight of fabric sample. The impregnated fabric was dried at $110^{\circ} \mathrm{C}$ for 3 minutes, then cured at $165^{\circ} \mathrm{C}$ for 2 minutes. It has to be pointed out that only the cotton component of the blend normally requires a resin finish.

The laundering is carried out under conditions similar to what the fabrics are expected in normal service. The Hoover Logic 1300 washer/dryer was used. The temperature was $40^{\circ} \mathrm{C}$. The detergent used was Bold Automatic washing powder with biological action and softening agents. This is one of the most popular detergents on the market. It contained, amongst other ingredients, less than $5 \%$ polycarboxylates cationic surfactants, nonionic surfactants and zeolites, 5-15\% anionic surfactants, oxygen-based bleaching agents, 15-30\% phosphates, enzymes and brightening agents. After washing and drying, the fabrics were allowed to lie flat and straight on a table for twenty-four hours in atmospheric conditions of $65 \pm 2 \% \mathrm{RH}$ and $20 \pm 2{ }^{\circ} \mathrm{C}$. Using the lowest setting on an iron, the fabrics were ironed, and prepared for testing.

The fabric tensile strength and elongation (warp and weft),

\footnotetext{
${ }^{*}$ Corresponding author Fax: +44-161-955-8159, E-mail: Hugh.Gong(a)UMIST.ac.uk
} 
shear strength (warp and weft), abrasion resistance, shrinkage (warp and weft), thickness and areal density were tested following 10, 20 and 30 launderings. Tensile properties were tested on an Instron machine according to BS2576:1967. Tear strength was tested on the SDL Elmendorf shear strength tester. Abrasion resistance was measured using the Martindale Abrasion Tester. The number of rubs to produce a hole in the fabric was used as a measure of resistance to abrasion.

To measure fabric shrinkage, samples of $35 \mathrm{~cm}$ by $11 \mathrm{~cm}$ dimensions were prepared before laundering. The effective length for shrinkage measurement was $30 \mathrm{~cm}$ under a pre-tension of $5 \mathrm{~g} / \mathrm{cm}$. The effective length was marked with wash-resistant markings. After laundering and drying, the fabric length between the markings was measured under the same pre-tension. Fabric shrinkage, expressed as the percentage changes in both warp and weft directions, was calculated according to the following formula:

$$
\text { Shrinkage }(\%)=100 \frac{L 1-L 2}{L 1}
$$

Where $\mathrm{L} 1=$ length before laundering $(30 \mathrm{~cm})$ and $\mathrm{L} 2=$ length after laundering.

The fabric thickness was measured using the FAST compression tester under $2 \mathrm{~g} / \mathrm{cm}^{2}$ (T2) and $100 \mathrm{~g} / \mathrm{cm}^{2}$ (T100) pressures. The fabric areal density was measured using a simple electronic balance.

\section{Results and Discussions}

The complex actions of laundering are expected to cause degradation of fabric performance. Changes in tensile strength are a good indicator of degradation. The tenacity of the all the fabrics becomes lower, especially in the warp direction of the crease resistant treated fabric, as the number of laundering cycles increases (Fig. 1). The warp direction exhibits higher strength than the weft direction. This is usually the case for most fabrics as the warp yarns are required to be stronger, but this is not an important issue for this investigation. The crease resistant treatment clearly reduces the fabric tensile strength as the processes of resin application and high heat curing and the acid formation caused by the presence of chlorine generally weakens the cotton fibres

As shown in Fig. 2, the fabric elongation at break increases sharply after the first ten laundering cycles and gradually stabilizes after that. This increase is caused by the relaxation during laundering as a result of the fabric being subjected to mechanical agitation under wet and dry conditions in a relaxed state. Fibre and yarn crimp increases by relaxation and consequently the fabric elongation increases. As the number of laundering cycle increases, the fabrics are in an increasingly more relaxed state, and therefore further laundering will bring about less and less changes. The crease resistant treatment reduces the fabric elongation in the weft direction. This reduction appears to be permanent because it remains almost constant after all laundering cycles. Crease resistant treatment sets-in the fibre and yarn crimp and also increases the inter-fibre and inter-yarn friction [15], it therefore reduces the fabric elongation. However, there is no noticeable influence in the warp direction, possibly due to the lower warp fibre and yarn crimp existing in the original fabric.

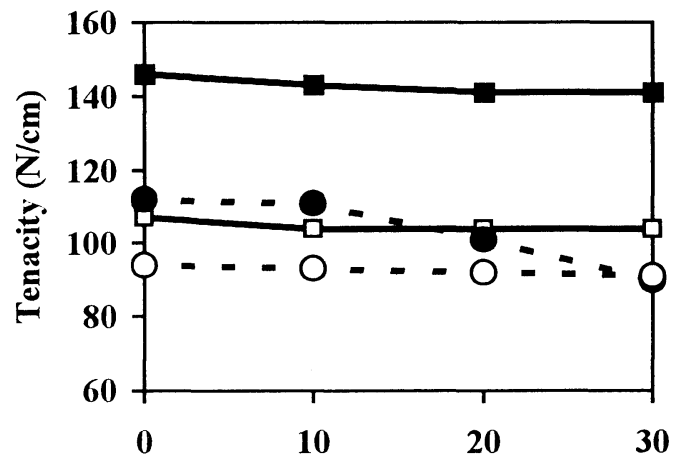

Laundering and drying cycles

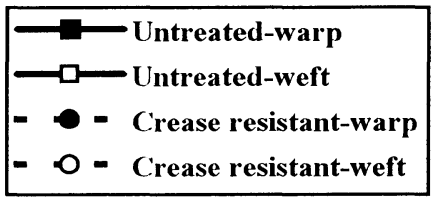

Fig. 1 Effects of laundering on tenacity

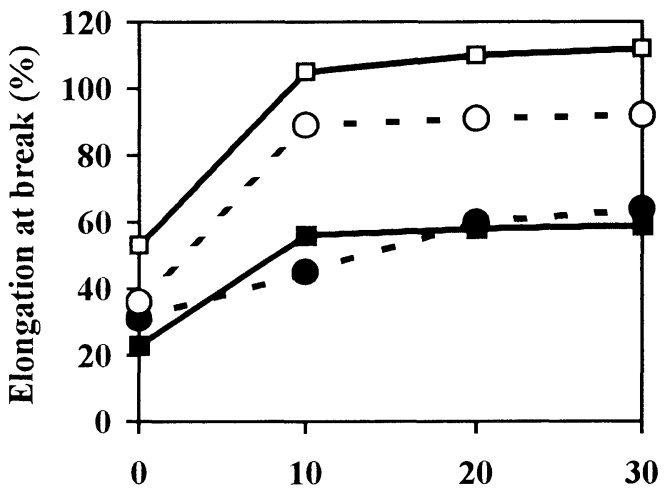

Laundering and drying cycles

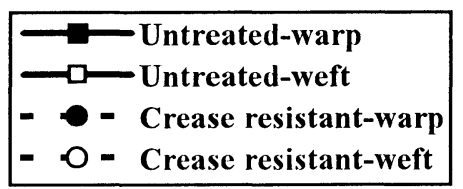

Fig. 2 Effects of laundering on break elongation 
The fabric tear strength follows similar trends to the tenacity (Fig. 3). This is to be expected because the tearing test essentially breaks the yarns. When testing warp tear strength, the weft yarns break; when testing weft tear strength, the warp yarns break. As the warp yarn is stronger than the weft yarn, the tear strength in the weft direction is higher than in the warp direction. In addition, because of the setting effect and the increased inter-yarn friction, less slippage can occur during tearing of the crease resistant treated fabric. This reduces the tear strength of the treated fabric.

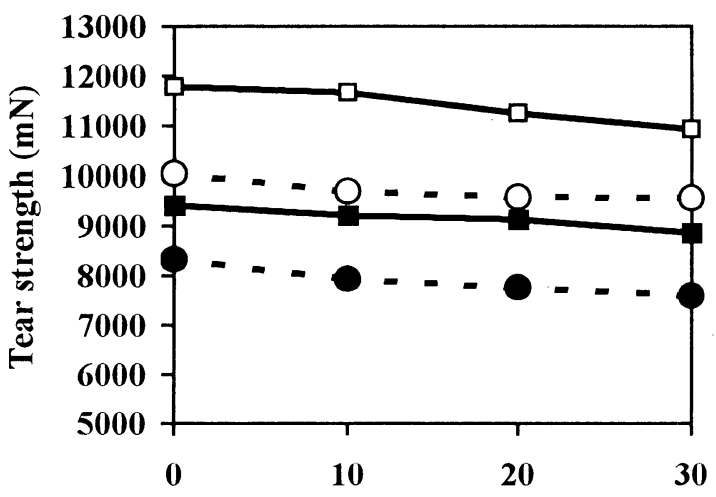

Laundering and drying cycles

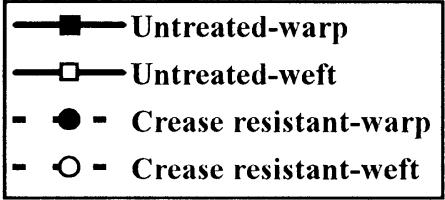

Fig. 3 Effects of laundering on tear strength

The fabric abrasion resistance increases sharply after the first ten laundering cycles, as shown in Fig. 4. Although it appears to be unusual at first sight, this can easily be explained by the shrinkage caused by the relaxation during laundering (Fig. 5). Shrinkage makes the fabric tighter, thinker (Fig. 6) and also heavier (Fig. 7). The tighter construction helps to hold the yarns in position and prevents fibres from being pulled out by rubbing, increasing the fabric's resistance to abrasion damage. It is to be expected that the thicker the fabric the longer it takes to wear, all other parameters being equal. The cotton fibre component in the fabric is an additional cause of shrinkage to fabric relaxation. The hygroscopic nature of cotton fibres causes them to swell when soaked in water. This swelling is accompanied by shrinkage in the fibre length. During drying, the reverse occurs, but the yarn cannot recover completely due to the frictional forces. Crease resistant treatment clearly improves the fabric stability as evidenced by the lower shrinkage. This is due to the aforementioned setting effect and the increased inter-fibre and inter-yarn friction. It is clear from Figures 4 and 5 that further increases in shrinkage after the first ten launderings do not increase the abrasion resistance to the same degree. This is because the effect of fabric shrinkage is reduced by the gradual decrease of fibre strength after increasing amount of laundering.

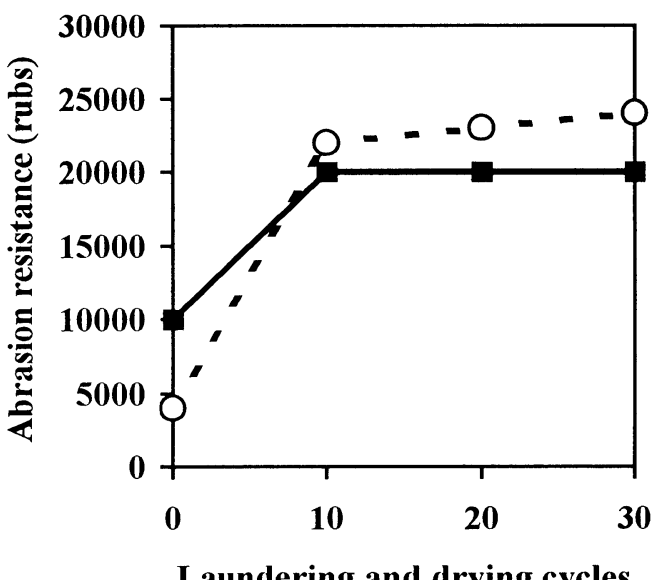

Laundering and drying cycles

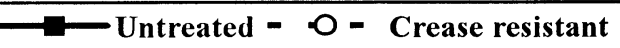

Fig. 4 Effects of laundering on abrasion resistance

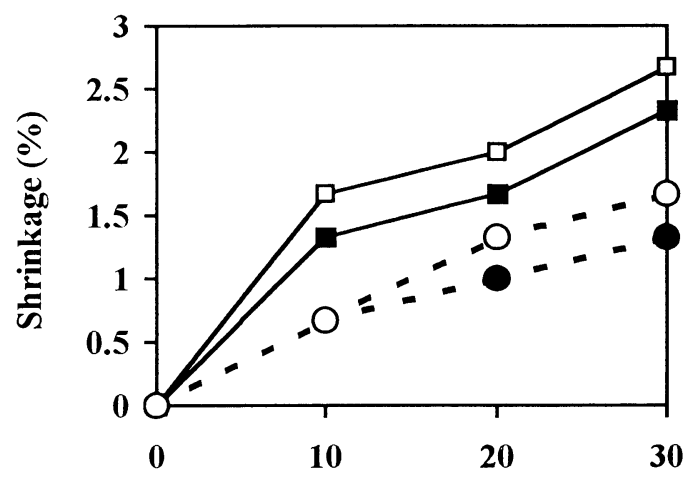

Laundering and drying cycles

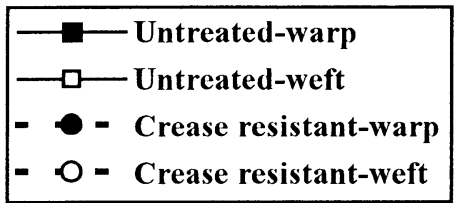

Fig. 5 Fabric shrinkage during laundering 

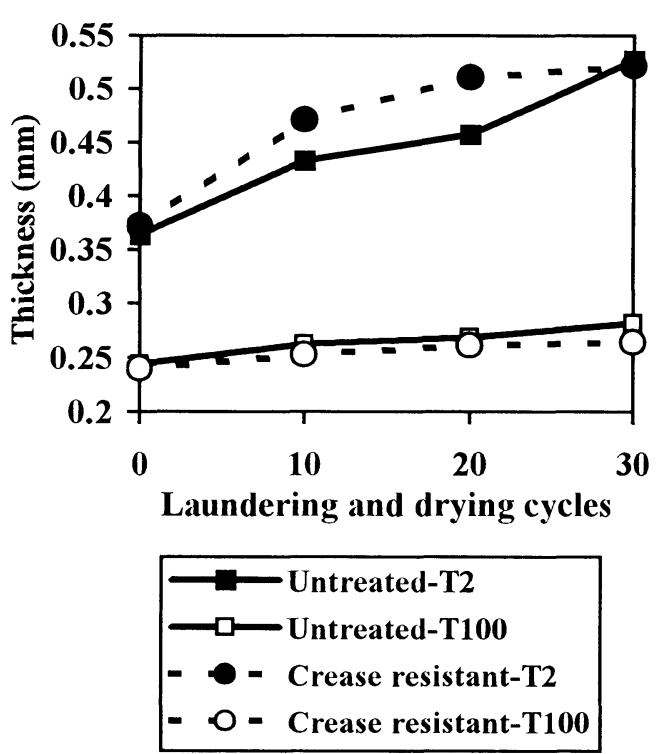

Fig. 6 Effects of laundering on fabric thickness

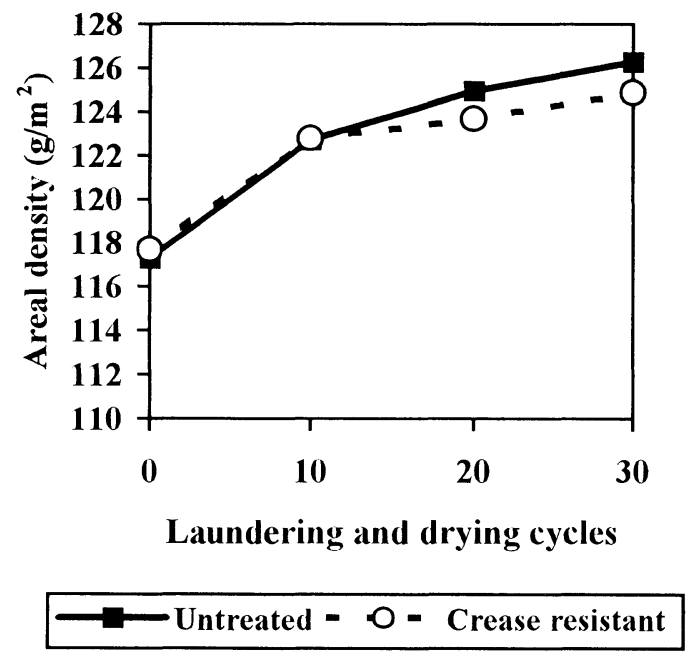

Fig. 7 Effects of laundering on fabric areal density

\section{Conclusions}

(1) Repeated laundering results in gradual reduction of fabric tensile and tear strength. This is due the degradation suffered by the fibres during laundering.

(2) The fabric elongation at break increases after repeated laundering, but the rate of this increase becomes lower as the fabric is subjected to more and more launderings. This is caused by the increased fibre and yarn crimp as the result of fabric relaxation during laundering.

(3) The fabric abrasion resistance increases after laundering. This is because the fabric becomes tighter, thicker and heavier caused by shrinkage during laundering. Fabric shrinkage occurs because of fabric relaxation and also the irrecoverable shrinkage of cotton fibres during repeated wetting and drying.

(4) As expected, crease resistant treatment improves the fabric stability, but this is achieved at the cost of reduced fabric strength.

The fabric abrasion resistance seems to be improved by crease resistant treatment, but only after laundering. This cannot be satisfactorily explained by the results obtained in this investigation. Further examination of the fabric including SEM analysis may help to understand this phenomenon.

\section{References}

[1] Murdison, M. E., Roberts, J. S., J. Text. Institute, 40, T505 (1949).

[2] Murdison, M. E., Roberts, J. S., J. Text. Institute, 41, T429 (1950).

[3] Vaeck, S. V., J. Soc. Dyers and Colourists, 83, p.374 (1966).

[4] Jacob, M., Subramanniam, V., Colourage, 33, No 8, p. 15 (1986).

[5] Verrall, M, Nature, 369, p.511 (1994).

[6] Markezich, A. R., Smith, M. M., Textile Chemist and Colorist, 3, No 6, p.140 (1971).

[7] Raheel, M. Lien, M. D., Text. Res. J., 52, p.493 (1982).

[8] Raheel, M. Lien, M. D., Text. Res. J., 52, p.555 (1982).

[9] Raheel, M. Lien, M. D., Text. Res. J., 53, p.557 (1983).

[10] Morris, M. A. Prato, H. H., Text. Res. J., 45, p.395 (1975)

[11] Sands, G. W., Canadian Text. J., 78, p.47 (1961).

[12] Standford, D. G., Georgouras, K. E., Pailthorpe, M. T., J. Euro. Academy of Dermatology and Venereology, 5, p. 28 (1995).

[13] Hall, A. J., The Standard Handbook of Textiles, Heywood Books, London, 1969.

[14] Trotman, E. R., Edward, R., Dyeing and Chemical Technology of Textile Fibres, Worcester, 1984.

[15] Taylor, H. M., J. Text. Institute, 50, T161 (1959). 